\title{
Study of Charge Diffusion in a Silicon Detector Using an Energy Sensitive Pixel Readout Chip
}

\author{
E. J. Schioppa, J. Idarraga, M. van Beuzekom, J. Visser, E. Koffeman, E. Heijne, K. J. Engel, and J. Uher
}

\begin{abstract}
A $300 \mu \mathrm{m}$ thick thin p-on-n silicon sensor was connected to an energy sensitive pixel readout ASIC and exposed to a beam of highly energetic charged particles. By exploiting the spectral information and the fine segmentation of the detector, we were able to measure the evolution of the transverse profile of the charge carriers cloud in the sensor as a function of the drift distance from the point of generation. The result does not rely on model assumptions or electric field calculations. The data are also used to validate numerical simulations and to predict the detector spectral response to an X-ray fluorescence spectrum for applications in X-ray imaging.
\end{abstract}

Index Terms - Charge sharing, diffusion, semiconductor detectors, timepix, x-ray imaging.

\section{INTRODUCTION}

$\mathbf{T}$ HE performance of a semiconductor particle detector is strongly affected by the charge transport properties of the sensor material. Diffusion, in particular, is a main concern in the case of thick, microscopically segmented devices, such as pixel detectors, since it gives rise to charge sharing phenomena, in which part of the charge created in a detector element is shared with a neighboring element. Recent imaging pixel devices can provide an energy measurement of the detected signal by measuring the amount of charge that the particle has released. This measurement can be accomplished at the level of a single pixel. In such cases, and especially for small pixel sizes, the presence

Manuscript received April 12, 2015; revised July 05, 2015; accepted August 27, 2015. Date of publication September 18, 2015; date of current version October 09,2015 . This work is part of a research program funded by the Foundation for Fundamental Research on Matter (FOM), which is part of the Netherlands Organization for Scientific Research (NWO). The research has been supported by the Dutch Technology Foundation (STW), which is the applied science division of NWO, and the Technology Programme of the Ministry of Economic Affairs, Project OTP 11956.

E. J. Schioppa was with Nikhef, Science Park 105, 1098 XG, Amsterdam, The Netherlands. He is now with Département de Physique Nucléaire et Corpusculaire (DPNC), Université de Genéve, Geneva, Switzerland (e-mail: enrico.junior.schioppa@cern.ch).

J. Idarraga was with NASA, USA. He is now with Nikhef, Science Park 105, 1098 XG, Amsterdam, Netherlands.

M. van Beuzekom, J. Visser and E. Koffeman are with Nikhef, Science Park 105, 1098 XG, Amsterdam, Netherlands.

E. Heijne is with Nikhef, European Center for Nuclear Research, Geneva, Switzerland (CERN) and the Institute of Experimental and Applied Physics (IEAP) of Czech Technical University, Prague, Czech Republic.

K. J. Engel is with Philips Research Eindhoven, Amsterdam, Netherlands.

J. Uher was with Amsterdam Scientific Instruments (ASI), Amsterdam, The Netherlands.

Color versions of one or more of the figures in this paper are available online at http://ieeexplore.ieee.org.

Digital Object Identifier 10.1109/TNS.2015.2475124 of charge sharing effects can seriously degrade the spectral resolution.

For a full characterization of the spectral response of an energy sensitive detector, the knowledge of the charge transport properties of the sensor is needed. In this work, we present a method that, thanks to the spectral sensitivity and high granularity of a hybrid semiconductor pixel detector, exploits a beam of highly energetic charged particles to measure directly the evolution of the cloud of charge carriers during its drift towards the collection electrode in a pixel detector. We apply the method to a silicon sensor connected to the spectroscopic pixel readout ASIC Timepix [1]. The analysis does not require knowledge of the electric field configuration in the sensor.

Charge sharing effects in Medipix and Timepix types of detectors have been extensively studied. Campbell et al. [2] studied the size of the pixel clusters generated by alpha particles of different energies. However, alpha particles are absorbed in the first few microns of the sensor, and do not allow for a study of bulk properties. A similar measurement based on the study of the pixel clusters size with different heavily ionizing particles was presented in [3]. Measurements performed using $\mathrm{X}$-ray radiation were presented as well, for example, in [4] using a strongly collimated beam, and in [5] using a Medipix 1 early prototype. In these published results, the actual interaction depth is not accurately known. These types of measurements are therefore not sufficient for a precise measurement of the charge diffusion profile in the full sensor volume. A possible solution is the scanning of the pixel volume area using a sub-pixel size narrow beam of synchrotron light, to study charge collection as a function of the position of the beam spot (for example, in [6]). This method allows, in fact, to measure precisely the evolution of the charge profile. However, precise scanning of the pixel volume requires complex mechanics, and the probing of the sensor is localized to a single or few pixels. In our method, we exploit tracks parallel or nearly parallel to the silicon sensor to retrieve information about the position of the passage of single particles within the pixel volume. An advantage of the proposed technique is that a large sensor area can be probed with high precision in a very simple experimental condition. Thanks to the geometry of the setup, the depth of interaction in each pixel is known with high accuracy and many points in the bulk of the sensor material which act as origins of the charge can be probed. In the data analysis, this information is correlated with the energy deposition measured by single pixels, from which the evolution of the charge profile as a function of the drift distance is extracted. 


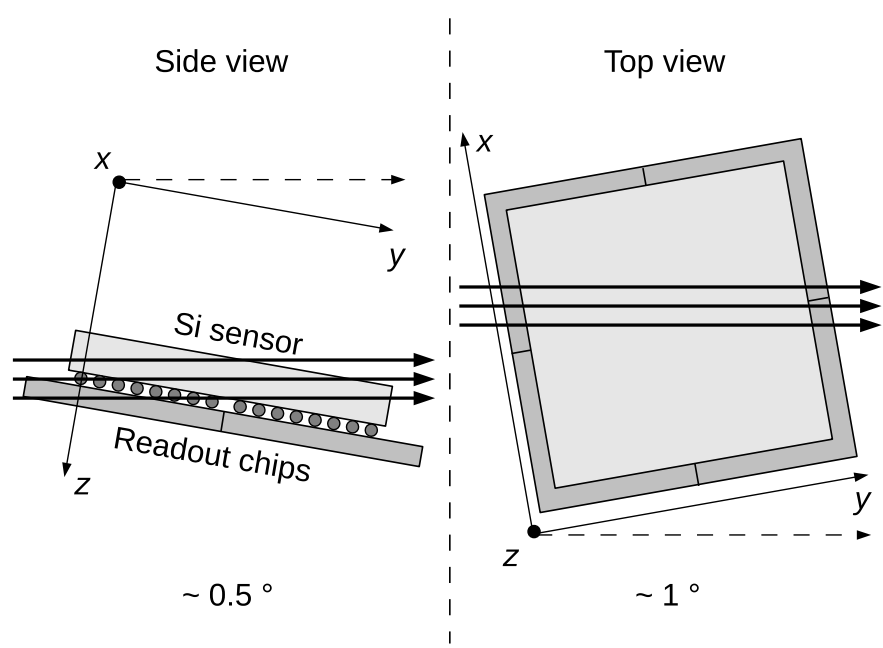

Fig. 1. Setup geometry: positioning of the detector in the beam. The beam direction is indicated by arrows. Also shown are the coordinate axes $(x, y, z)$ defining the reference frame used in the analysis.

TABLE I

Most Relevant Sensor Properties

\begin{tabular}{|l|l|}
\hline Producer & VTT, Finland \\
\hline Material & FZ $\langle 100\rangle \mathrm{n}$-type Silicon \\
\hline Sensor type & p-on-n \\
\hline Thickness & $300 \mu \mathrm{m}$ \\
\hline Full depletion voltage & about $40 \mathrm{~V}$ \\
\hline Resistivity & about $7 \mathrm{k} \Omega \mathrm{cm}$ \\
\hline Implantation & $\begin{array}{l}\text { about } 1 \mu \mathrm{m} \text { deep for both the pixels (pn- } \\
\text { junction depth) and cathode }\end{array}$ \\
\hline Metallisation & $500 \mathrm{~nm}$ of AlSi $(1 \%$ Si) on both sides \\
\hline Passivation & $500 \mathrm{~nm}$ of Low Temperature Oxide LTO \\
\hline
\end{tabular}

\section{EXPERIMENTAL SETUP}

A $300 \mu \mathrm{m}$ thick p-on-n silicon sensor was bump-bonded to a $2 \times 2$ matrix of 4 Timepix chips, for a total of $512 \times 512$ square pixels, of $55 \mu \mathrm{m}$ size, on an area of approximatively $2.8 \times$ $2.8 \mathrm{~cm}^{2}$. The sensor was biased at $100 \mathrm{~V}$ during all measurements. A list of the most relevant characteristics of the sensor is given in Table I.

The data acquisition was performed using the $1 \mathrm{~Gb}$ Ethernet RelaxD [7] system. Data were collected from a beam of $40 \mathrm{GeV}$ negative pions at the H6-SPS experimental area at CERN.

Fig. 1 shows the geometry of the setup. The sensor was positioned in the beam such that particles crossed it longitudinally. Such geometry is sometimes referred to as 'glancing (or grazing) angle' or 'edge-on' direction by other authors. A small tilt $\left(\sim 0.5^{\circ}\right)$ in the polar angle was applied in order for a charged particle to leave a long straight track (200 pixels on average) that, at the same time, has a high probability to be contained within the detector area. Another small tilt $\left(\sim 1^{\circ}\right)$ was applied in the detector plane to ensure that tracks would span different pixel rows. In such a configuration, the detected particle tracks can be fitted with a straight line and the resulting parameters can be used to achieve sub-pixel precision on the positions where the particle has passed inside the pixel volume, which determine the points of charge generation along the particle trajectory.

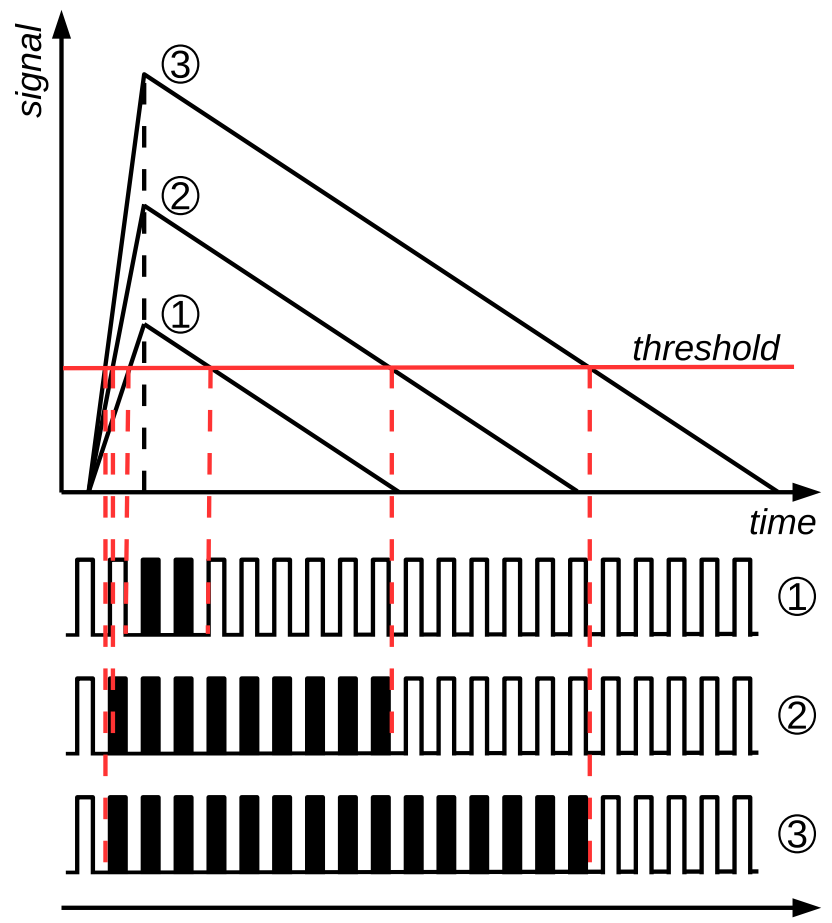

clock

Fig. 2. ToT measurement in Timepix.

\section{Per-Pixel Energy Calibration}

The energy measurement in the Timepix chip is based on counting the cycles of a high-frequency (e.g., $40 \mathrm{MHz}$ ) clock for as long as a signal stays above threshold. This mode of operation is called ToT (time-over-threshold) and, in essence, it corresponds to the measurement of the integral charge signal using a Wilkinson-type Analog to Digital Converter (ADC). The pixel front-end of the Timepix chip implements a charge sensitive amplifier and a shaper stage where the collected charge is shaped into a pulse whose duration is proportional to the charge, therefore to the energy deposition. This situation is represented in Fig. 2, where the ToT measurement is shown for three different pulses generated by an increasingly higher charge. The typical energy resolution achieved with the Timepix ToT method is in the order of $10-15 \%$ [8], [9]. Notice in Fig. 2 that the method introduces timewalk effects. A new version of the Timepix chip, called Timepix 3 , has recently been designed which addresses this effect by simultaneously measuring ToT and Time of Arrival (ToA) [10].

A ToT-to-energy calibration of single pixels was performed using an Fe-55 source as well as X-ray fluorescence emission from pure metal targets irradiated by an X-ray tube. This calibration has been used to convert the ToT values in the test beam data to energy. An example of a ToT calibration plot for a single pixel is shown in Fig. 3. The data points are fitted according to the method described in [11], accounting for the typical loss in linearity at low energies, which is due to the electronics design.

Although a precise measurement of energy deposition in our sensor is not the goal of this work (the results on diffusion we present are independent on absolute energy measurements), it is still interesting to make a few considerations about the measured 


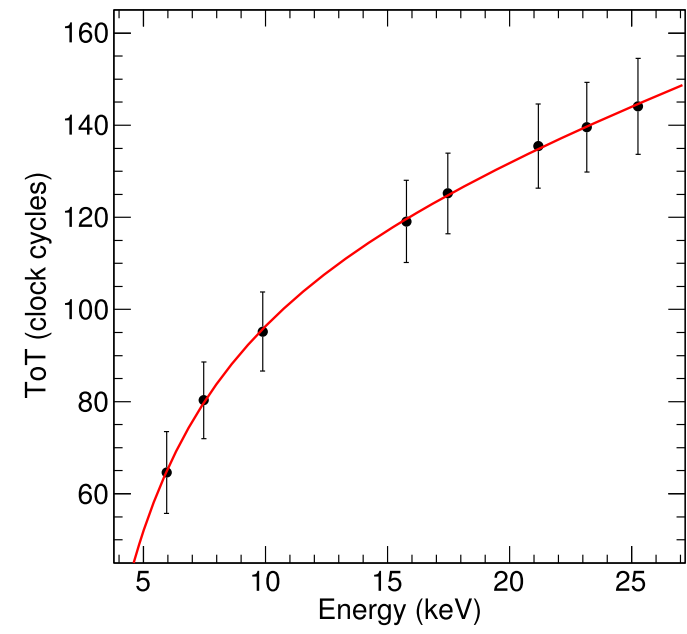

Fig. 3. ToT to energy calibration of a single pixel.

values. The restricted energy loss formula for high energy pions and muons in silicon predicts that electron-hole pairs be created with a linear density of $75 \mu \mathrm{m}^{-1}$ when traversing a $300 \mu \mathrm{m}$ thick sensor [12]. In our case, due to the grazing angle geometry, the calibrated energy values measured at each pixel correspond to the energy deposited along the longitudinal $55 \mu \mathrm{m}$ path through the pixel volume and the pair density is expected to be lower, around $67 \mu \mathrm{m}^{-1}$. This is well illustrated in Fig. 4, showing the distribution of the average per-pixel energy deposition we obtain from our data. The distribution is fitted via a Landau curve convoluted with a Gaussian $-\chi^{2} / n d f=40.72 / 42$ - (see Section VI-C), yielding a most probable value (MPV, unless otherwise specified, in this paper, we will always refer to energy loss in terms of most probable value rather than mean value) for the energy deposition of $12.81 \pm 0.07 \mathrm{keV}$, corresponding to a $64.69 \pm 0.35 \mu \mathrm{m}^{-1}$ pair density (having used $3.6 \mathrm{eV}$ for the average energy required to create an electron-hole pair in silicon). The slight difference from the expected value can be attributed to multiple causes. Effects that can lead to a systematically lower measurement of energy deposition are charge sharing and systematic errors in the calibration of the Timepix ToT, especially in such low energy region.

An interesting point worth stressing is how our track selection (discussed inSection IV) influences the measured energy deposition. A key feature of the analysis is the removal, from the set of track hits, of those corresponding to the emission of delta rays. It will then turn out that the MPV of the energy deposition shifts down to around $11 \mathrm{keV}$, corresponding to a $55.5 \mu \mathrm{m}^{-1}$ pair density, showing that delta rays contribute for almost $15 \%$ to the total energy deposition.

\section{Data Selection}

Different interactions that a particle can undergo with the sensor material give rise to different classes of events. Fig. 5 shows a set of typical ones. Each event in the figure consists of a cluster of particle hits where each dot represents a pixel with a non-zero digitized value. The gray scale color scale in the picture encodes the signal amplitude, namely the ToT value, of each pixel (or hit). In each cluster, a gap of maximum 3 empty

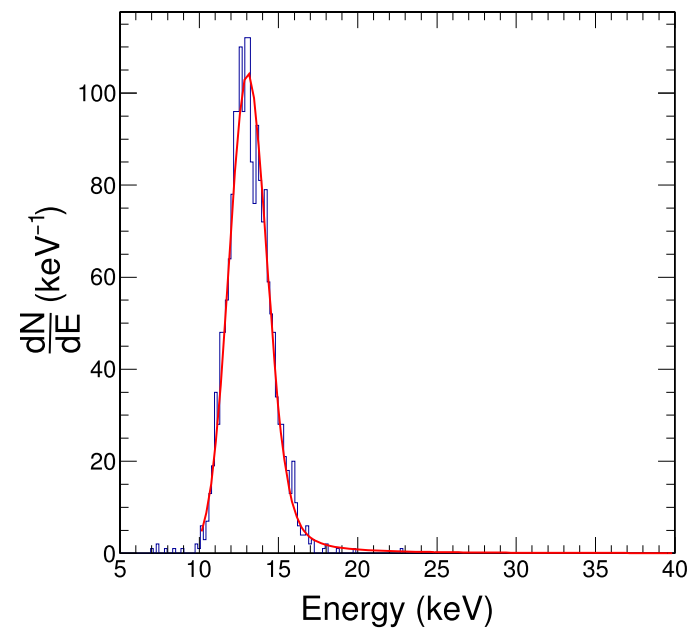

Fig. 4. Per-pixel average energy deposition prior to the processing of the track hits data.

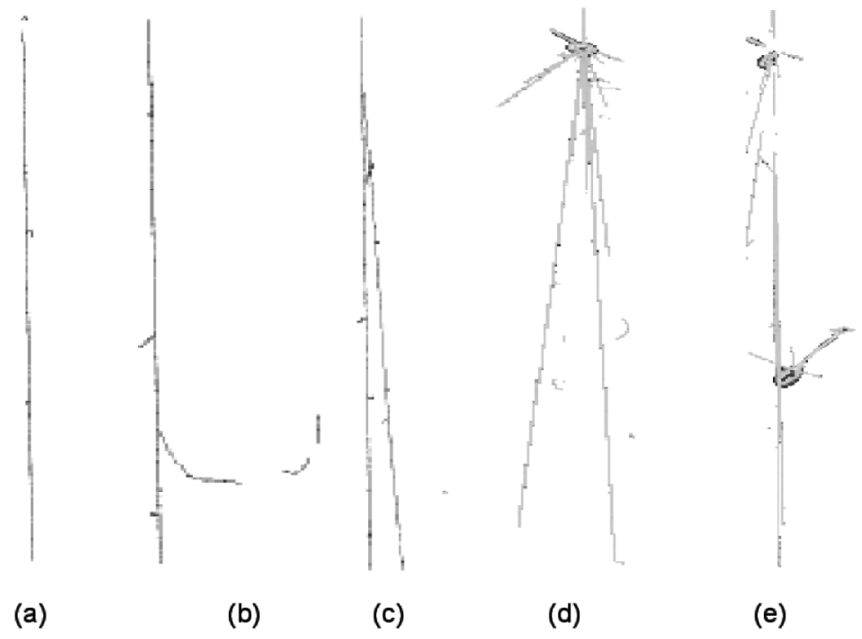

Fig. 5. Some examples of the typical track topologies: (a) a straight track from a charged particle with few delta electrons, (b) a highly energetic delta emission, (c) production of a high energy secondary particle, and (d)-(e) events with nuclear interactions.

pixels is allowed, in order to account for dead pixels or pixels where the signal has fluctuated below the threshold.

Two of the clusters in the figure (clusters (d) and (e)) present clear signatures of nuclear interactions. These type of events will be removed from the analysis, whereas straight tracks left by high energy primary particles, such as (a), are selected. Track selection is carried out according to the following requirements:

- The entry and exit points of a cluster must be fully contained within the detector area; clusters that contain pixels at the edge of the detector matrix are rejected.

- The track length $L$ (distance between first and last hits in the cluster) must be between 150 and 300 pixels.

- For each cluster, the

$$
\eta=\frac{\sqrt{A}}{L} .
$$

parameter is calculated. Here, $A$ is the area of the cluster, calculated by counting the pixels belonging to the cluster, 
but defined so that a single line cluster has $A=0$ (hence $\eta=0)$ rather than $A=L(\eta=1 / \sqrt{L})$. With this choice, the separation capability between straight tracks $(\eta$ close to zero) and clusters where nuclear interactions are present ( $\eta$ significantly larger than zero) is enhanced. In fact, if $L_{1}$ and $L_{2}$ are the number of transverse pixels by which two clusters of the same length $L$ deviate from being perfectly linear, the corresponding areas, according to our definition, are $A_{1}=L_{1}$ and $A_{2}=L_{2}$, thus yielding a separation $\Delta \eta$ $=\left|\eta_{2}-\eta_{1}\right|=\left|\sqrt{L_{2}}-\sqrt{L_{1}}\right| / L$. If the other definition would be taken ( $A=L$ for single line clusters), the separation would be lower: $\Delta \eta=\left|\sqrt{L_{2}+L}-\sqrt{L_{1}+L}\right| / L$. An example of the distribution of the $\eta$ values is shown in Fig. 6. In order to select straight tracks against events with the possible presence of a nuclear interaction, clusters with $\eta>0.05$ are rejected.

- A topological analysis is performed on each cluster in order to identify whether a pixel hit belongs to the main straight track or to a peripheral trail caused by a delta electron. The analysis is based on the assumption that sub-clusters originating from delta electrons mostly develop transversely to the main track. Each hit is analyzed individually to check whether it belongs to a mainly horizontal or vertical section of the cluster, and to check if it does not lay further than one pixel $(55 \mu \mathrm{m})$ far from a line fitting the main track. Hits that, according to this criteria, are associated with a delta electron are removed from the cluster. The topological analysis and the fit are repeated iteratively until the track is completely cleared of the delta electrons (see Fig. 7 for an example).

- Tracks surviving the selection criteria are fitted with a straight line using a linear least squares method. To minimize the correlation between the line parameters, the fit is performed in a reference frame where the origin corresponds to the geometric center of the track. The root mean square distance (RMSD) of the track hits to the fitted line is calculated and tracks with RMSD $>27.5 \mu \mathrm{m}(0.5$ pixels) are rejected (see Fig. 8).

Due to the geometrical gap between adjacent Timepix chips, the pixels that are located at the boundary between two chips are 3 times as large than the others ( 9 times for the 4 central pixels). Pixel coordinates of hits are therefore recalculated for all the clusters spanning two or more chips. The energy value of larger pixels are renormalized accordingly.

\section{Determination of Track Hit COORdinates}

For the selected tracks, the three coordinates of the space point of passage in each pixel ("hit" position) are determined. The $(x, y, z)$ axes of the reference frame are represented in Fig. 1: $x$ and $y$ are defined as the coordinates of the pixel rows and columns, while $z$ represents the coordinate in the sensor depth. The $y$ direction is chosen as the direction of main longitudinal development of the particle tracks, while $x$ is the (almost) transverse direction. The sensor backside corresponds to $z=0 \mu \mathrm{m}$, while the readout side corresponds to $z=300 \mu \mathrm{m}$.

Although the physical pixel size is $55 \times 55 \times 300 \mu \mathrm{m}^{3}$, the hit position in the track can be determined with much better preci-

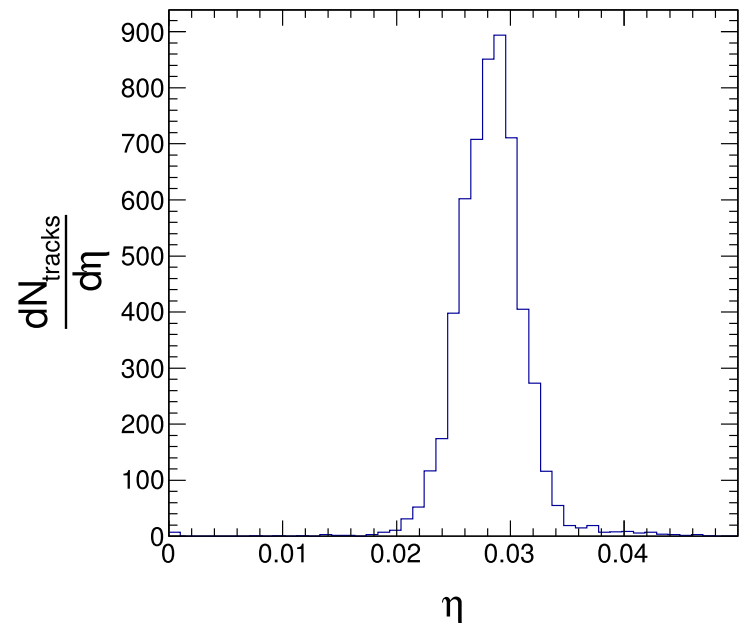

Fig. 6. Distribution of the $\eta$ parameter, that is a measure of the lateral extension of a 2-dimensional cluster of pixels.

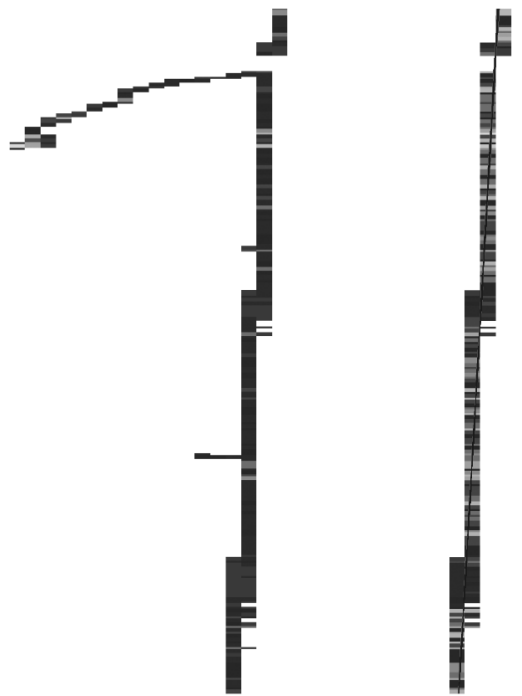

Fig. 7. An example of a charged particle track before (left) and after (right) the topological analysis and the fit. All the track hits that most likely belong to the trail of a delta electron are removed.

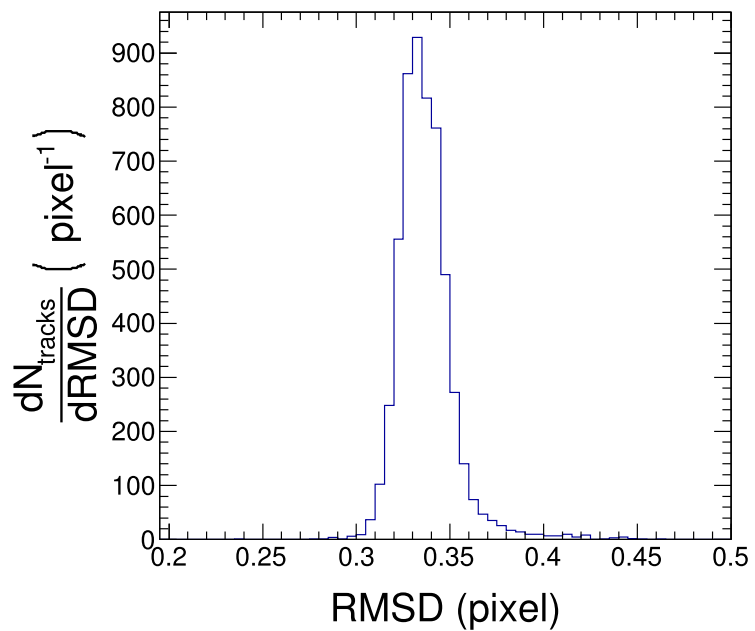

Fig. 8. Distribution of the root mean square distance (RMSD) of the pixel hits to the fitted track.

sion by interpolation, thanks to the grazing angle geometry. The $x$ coordinate of the pixel hit is calculated as the distance of the 


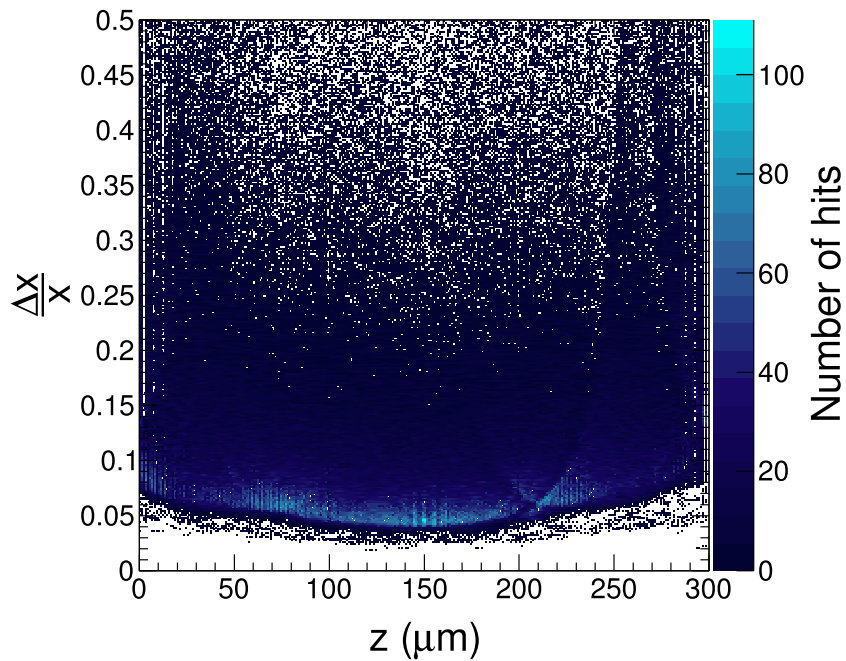

Fig. 9. Resolution $\Delta x / x$ for the $x$ coordinate of the track hits, as a function of the depth in the sensor $z$. In most cases, $\Delta x$ is in the order of few microns.

fitted track from the center of the pixel. As Fig. 9 shows, the distribution of the uncertainty $\Delta x / x$, determined by propagating the errors from the line fit parameters, peaks below $5-10 \%$.

The $z$ coordinate is given by

$$
z=t \frac{l}{L}
$$

where $t$ is the sensor thickness $(300 \mu \mathrm{m})$ and $l$ is the distance of the current hit from the first track hit. For an average track length $\mathrm{L}=200$ pixels, this yields a sub-micron uncertainty. This result is obtained by propagating the error in formula 2 , which yields

$$
\Delta z=\frac{t}{L^{2}} \sqrt{L^{2} \Delta^{2} l+l^{2} \Delta^{2} L}
$$

where $t=300 \mu \mathrm{m}$ is assumed as a parameter. Both $l$ and $L$ are calculated as the distance between two pixels, the first and the last in the case of $L$. Given a pixel size of $55 \mu \mathrm{m}$, this means that $\Delta l=\Delta L=55 / \sqrt{6} . \Delta z$ is bound by the extreme values assumed when $l=0$ and when $l=L$, which yield

$$
\frac{55 t}{\sqrt{6} L}<\Delta z<\frac{55 t}{\sqrt{3} L}
$$

For an average track length of 200 pixels, i.e., $L=200 \times 55 \mu \mathrm{m}$, this results in $0.61 \mu \mathrm{m}<\Delta z<0.87 \mu \mathrm{m}$.

\section{Analysis of LATERAL Charge DifFusion}

After track selection and track fitting, the complete data set consists of 5522 tracks and a total of more than 1 million pixel hits, each identified by a coordinate-energy vector $(x, y, z, E)$, where $E$ corresponds to the calibrated ToT value of the pixel. Due to the high sub-pixel precision with which the hit coordinates are known, these data allow to study how charge is collected in the sensor at different depths and at different positions in the pixel area.

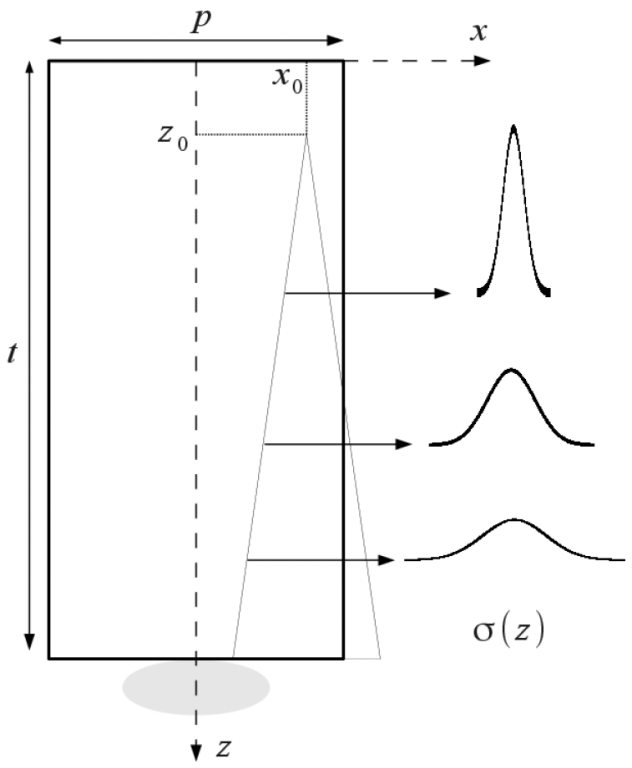

Fig. 10. Model of charge diffusion and charge sharing: the cloud is distributed according to a Gaussian profile in the $x$ direction, whose width $\sigma(z)$ increases as the carriers drift towards the collection electrode (at the bottom). The model is fully symmetrical for translations along the $y$ direction.

In order to avoid inter-chip mismatches, the analysis is carried out separately for each chip. The results shown refer to chip 0 (chip 0 and chip 3 were exposed to the highest beam intensity). For this chip the data set consists of 3981 tracks and more than half million good hits.

For each track, the two hits corresponding to the entry and exit points are not considered in the following analysis because, since these pixels are not fully traversed by the particle, their energy value is intrinsically uncertain.

\section{A. Charge Transport Model}

Fig. 10 shows the simple scheme employed to model charge transport. The sensor has thickness $t$ and is segmented into square pixels of size $p$. A fast ionizing particle enters the pixel at position $\left(x_{0}, z_{0}\right)$ and deposits energy along the $y$ direction through the $55 \mu \mathrm{m}$ path length, giving rise to an elongated cloud of charge carriers.

By treating carrier transport as a combination of drift and thermal diffusion only, the transverse (namely, along $x$ ) cloud profile evolution can be worked out starting from the general solution to Fick's law, namely

$$
\frac{d Q}{d x}(\vec{x}, t)=\frac{1}{8(\pi D t)^{3 / 2}} \int Q_{0}\left(\vec{x}^{\prime}\right) e^{-\frac{\left(\vec{x}-\vec{x}^{\prime}\right)^{2}}{4 D t}} d \vec{x}^{\prime}
$$

describing the general 3-dimensional distribution of the charge carriers at time $t$, given an initial distribution $Q_{0}\left(\vec{x}^{\prime}\right)$. In this formula, both the diffusion constant $D$ and the time dependence can be hidden by defining a generic Gaussian spread $\sigma$ $=\sqrt{2 D t(z)}$ that depends on $t$ through the inverse of the drift equation of motion $z(t)$. In principle, an expression for $z(t)$ is needed in order to calculate the $z$ component of the integral in 5 . This would require the full characterization of the electric field 
configuration in the sensor, from which one could then calculate the drift velocity field, to be integrated to yield $z(t)$. However, in the analysis that follows, what matters is not the time evolution of the charge cloud, but rather its dependence on the depth $z$ in the sensor. The key parameter is thus $\sigma(z)$ rather than $\sigma(t)$. A workaround to isolate $\sigma(z)$ from the integral in equation (5) is to assume that the charge distribution is initially point-like for a point-like particle interaction. In the case of our pion data, where deposition takes place along the full straight trajectory of the particle in the sensor, we can assume an elongated initial charge distibution along the $y$ direction of the form

$$
Q_{0}\left(\vec{x}^{\prime}\right)=\frac{d Q_{0}}{d y} \delta\left(x^{\prime}-x_{0}\right) \delta\left(z^{\prime}-z_{0}\right)
$$

for a linear density $\frac{d Q_{0}}{d y}$ along $y$. By using this expression, equation (5) yields

$$
\frac{d Q}{d x}\left(x, z ; x_{0}, z_{0}\right)=\frac{Q_{0}}{\sqrt{2 \pi} \sigma\left(z, z_{0}\right)} e^{-\frac{\left(x-x_{0}\right)^{2}}{2 \sigma^{2}\left(z, z_{0}\right)}},
$$

where now $Q_{0}$ is the total charge generated by the particle in this pixel.

The results of the analysis will show that, in fact, the initial size of the charge cloud, as determined by fitting this model to the data, is not negligible. The ratio $\sigma\left(z=300 \mu \mathrm{m}, z_{0}=\right.$ $300 \mu \mathrm{m}) / \sigma\left(z=0 \mu \mathrm{m}, z_{0}=300 \mu \mathrm{m}\right)$ between the final and initial cloud spread after drifting through the full $300 \mu \mathrm{m}$ sensor depth, is in the order of $10 \%$. A systematic study on the effect of the initial point-like approximation must be addressed in future work. In particular, the study presented in [13] on the energy dependence of the initial cloud size could also benefit from such results.

\section{B. Charge Sharing Model}

At the level of the present analysis, we will work assuming that expression 6 is the initial distribution of charges. Under this assumption, we can derive a simple formula for the charge collected by the pixel as a function of the position where the pion has passed through its volume. Such a formula will only depend on one parameter, i.e., the final cloud spread $\sigma\left(300, z_{0}\right)$ as a function of the initiation depth $z_{0}$, which can thus be easily retrieved by fitting the data. Furthermore, the model is now independent of both the transport properties of the sensor (no parameters such as the diffusion constant and the carrier mobilities appear explicitly) and the electric field configuration (since the time dependence is hidden).

The only assumption we make about the electric field is that it is parallel through all the sensor depth, i.e., we neglect the field distortions occurring in the vicinity of the pixelated electrodes. This assumption does not affect the results we are about to derive. The effect is, in fact, systematic and one can think of the results as related to an "effective" linear field which, excluding changed bulk properties of the sensor due to, e.g., radiation effects, has no reason to be different between different operations of the detector under the same conditions. If we also assume that no charge is lost during the drift process (which our data will show later to be, in fact, the case), the charge collected by

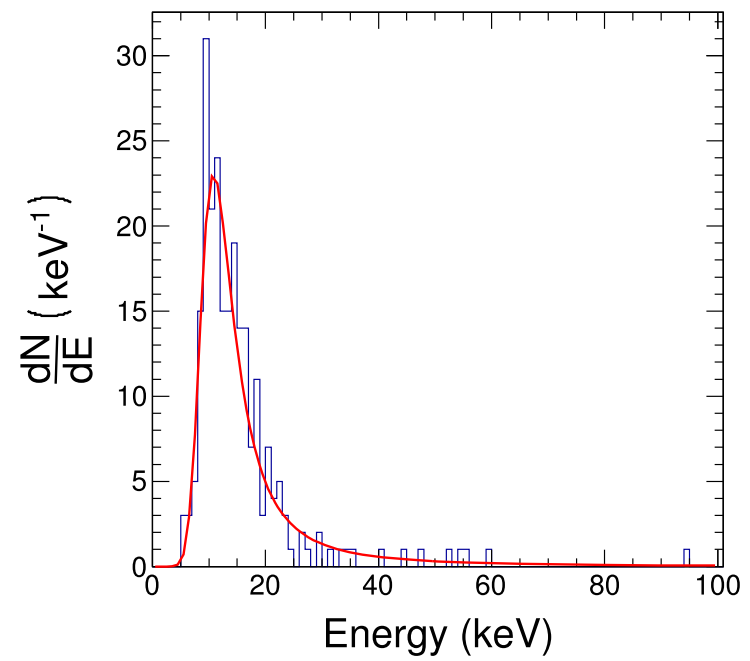

Fig. 11. Landau curve fitting the energy distribution of track hits at a specific $\left(x_{0}, z_{0}\right)$ bin.

the electrode is the integral of the charge density in the pixel area at depth $z=300 \mu \mathrm{m}$, namely

$$
Q\left(x_{0}, z_{0}\right)=\int_{-p / 2}^{p / 2} \frac{d Q}{d x}\left(x, 300 ; x_{0}, z_{0}\right) d x .
$$

If $\sigma\left(z, z_{0}\right)<p / 2$ for all values of $z$ and $z_{0}$, and in the situation in Fig. 10 where $x_{0}>0$ (the negative $x_{0}$ case can be treated in an analogous way) the previous expression can be rewritten as

$$
Q\left(x_{0}, z_{0}\right) \sim \frac{Q_{0}}{2}+\int_{x_{0}}^{p / 2} \frac{d Q}{d x}\left(x, 300 ; x_{0}, z_{0}\right) d x .
$$

Using 7, this expression can be worked out to yield

$$
Q\left(x_{0}, z_{0}\right) \sim \frac{Q_{0}}{2}\left[1+\operatorname{erf}\left(\frac{p / 2-x_{0}}{\sqrt{2} \sigma\left(300, z_{0}\right)}\right)\right]
$$

The collected charge $Q$ is proportional to the energy released by the particle in the $55 \mu \mathrm{m}$ path in the silicon pixel. It therefore fluctuates according to a Landau distribution with $Q_{0}$ as most probable value. This formula can thus be used to fit the data in order to retrieve the values of $\sigma\left(300, z_{0}\right)$ for different depths $z_{0}$.

\section{Determination of $\sigma\left(z_{0}\right)$}

The track hit positions were binned in $1 \mu \mathrm{m}$ intervals in $z_{0}$ and $1.5 \mu \mathrm{m}$ intervals in $x_{0}$ from $x_{0}=-75 \mu \mathrm{m}$ to $x_{0}=+75 \mu \mathrm{m}$. At each $\left(x_{0}, z_{0}\right)$ bin, a plot of the energy distribution is made. A Landau curve convoluted with a Gaussian profile [14][15], times an error function modeling the onset of the detector threshold at $4.50 \mathrm{keV}$, was fitted to each distribution, and the most probable value for the energy depositionloss was retrieved from the Landau component. A typical example is shown in Fig. 11 (most probable energy loss $10.53 \pm 0.26 \mathrm{keV}, \chi^{2} / n d f$ $=24.98 / 31)$.

The dataset is now reduced to a $100 \times 300$ matrix of energy values at the corresponding positions in the $\left(x_{0}, z_{0}\right)$ pixel plane. By plotting the most probable energy as a function of 


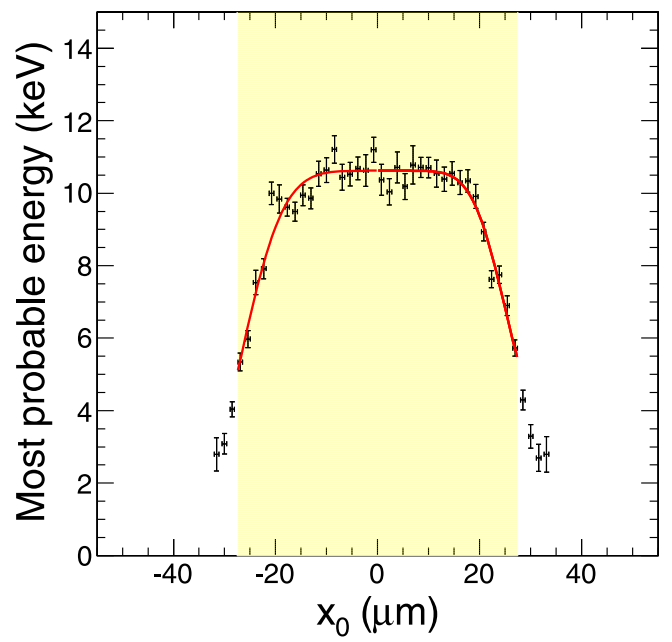

Fig. 12. Most probable energy, as obtained from the fit in Fig. 11, versus $x_{0}$, for $z_{0}=(76.5 \pm 0.9) \mu \mathrm{m}$. The central band highlights the pixel volume.

the $x_{0}$ coordinate at a given $z_{0}$ bin, the effect of charge sharing is immediately visible. Fig. 12 shows an example for $z_{0}=$ $(76.5 \pm 0.9) \mu \mathrm{m}$, where the central band in the plot highlights the pixel volume. The energy values at the center of the pixel are distributed on a plateau at around $10-11 \mathrm{keV}$, while they decrease more and more as the tracks approach the region outside the pixel, eventually dropping to half the plateau energy at the boundary between two pixels, and going towards zero when $\left|x_{0}\right|>p / 2$. The solid line shows the fit to the data according to the function defined in equation (10) and the corresponding one for $x_{0}<0$. The fit allows to extract the value of $\sigma\left(z_{0}\right)$ simultaneously from the left side (negative $x_{0}$ ) and from the right side (positive $x_{0}$ ). A mean value can be computed by averaging the two.

Notice that the total charge $Q_{0}$ appearing in equation (10) can be determined before the fit, by separately fitting a constant value to the plateau region of the plot in Fig. 12. As a consequence, the only free parameter in equation (10) remains $\sigma\left(z_{0}\right)$.

The same plot was made for each of the $300 z_{0}$ bins, and the fit was repeated for each distribution. As a result, the full $\sigma\left(z_{0}\right)$ function could be determined, as shown in Fig. 13.

\section{Discussion}

Fig. 14 shows, as a function of $z_{0}$, the most probable value of the energy released at small distances from the pixel center $\left(x_{0}\right)$, namely at the plateau region in the plots of the type shown in Fig. 12. The plot shows that these values are constant throughout all the sensor depth, with the only exception of a shallow region close to $z_{0}=300 \mu \mathrm{m}$, where charge collection inefficiencies are expected due to the distortion of the electric field close to the pixelated electrode. These data allow to conclude that (a) a charge cloud initiated in the center of the pixel is fully contained in the same pixel throughout the whole process of drift towards the electrode, and (b) all the charge that is created is collected. These conditions correspond, respectively, to the $\sigma\left(z, z_{0}\right)<$ $p / 2$ approximation and to the assumption that no charge is lost during the drift, thus validating the derivation of equation (10).

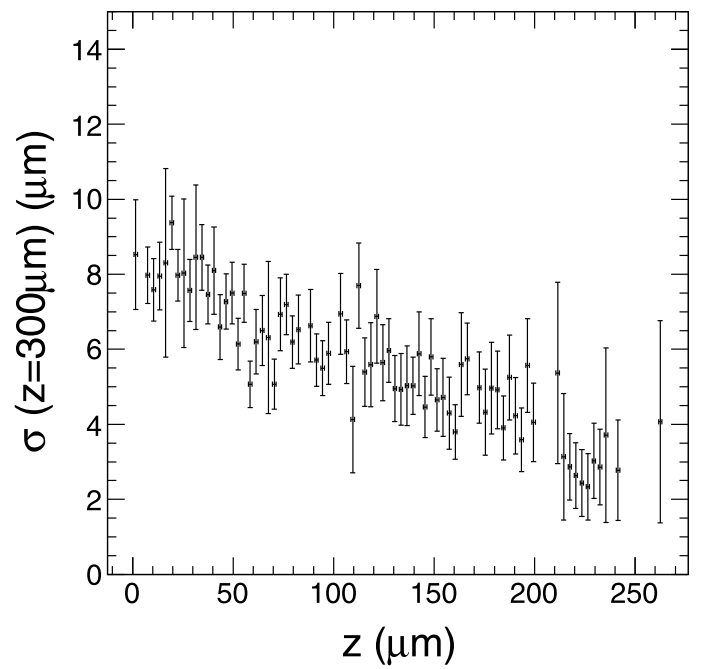

Fig. 13. Width of the Gaussian charge cloud profile in $x$ as a function of the drift distance.

A simple calculation can be carried out to validate the consistency of the measured values for the diffusion profile with expectations. The average electric field along the sensor full thickness $t=300 \mu \mathrm{m}$ at bias voltage $V_{b}=100 \mathrm{~V}$ is

$$
\bar{E}=\frac{1}{t} \int_{0}^{t} E(z) d z=\frac{V_{b}}{t},
$$

independently of the exact functional expression of $E(z)$. Defining an average drift velocity $\overline{v_{d}}=\mu \bar{E}=\mu V_{b} / t$ in terms of the carriers mobility $\mu$, the related drift time would be

$$
\text { time }=\frac{t^{2}}{\mu V_{b}},
$$

which would give a diffusion width

$$
\sigma=\sqrt{\frac{2 D t^{2}}{\mu V_{b}}} .
$$

Using the typical values for holes in silicon of the diffusion constant $\left(D=12 \mathrm{~cm}^{2} / \mathrm{s}\right)$ and the mobility $\left(\mu=450 \mathrm{~cm}^{2} / \mathrm{sV}\right)$, one obtains $\sigma=6.93 \mu \mathrm{m}$, in fairly good agreement with the result shown in Fig. 13.

Notice that formula 13 can be generalized by using Einstein's relation

$$
D=\frac{k_{B} T}{e} \mu,
$$

where $k_{B}$ is the Boltzmann's constant, $e$ is the elementary charge, and $T$ is the absolute temperature. In such a case, one has

$$
\sigma=\sqrt{\frac{2 k_{B} T}{e V_{b}} t^{2}}
$$




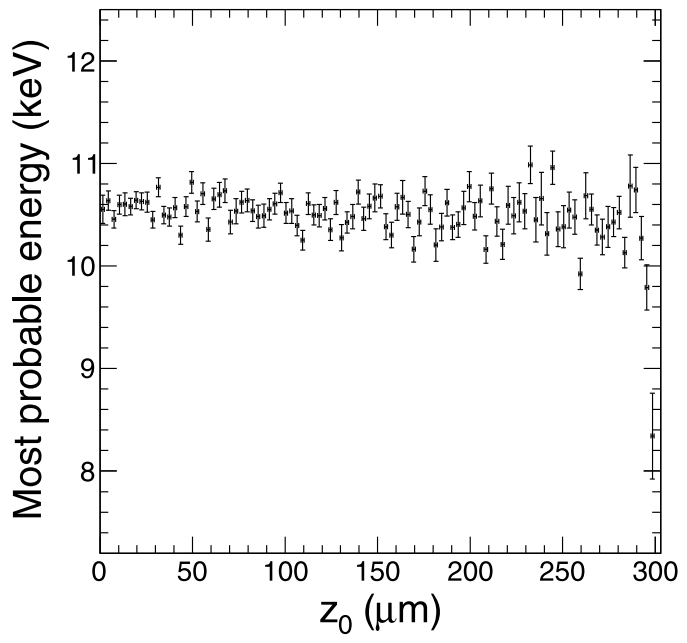

Fig. 14. Most probable value of the energy deposition at small distances from the pixel center $\left(x_{0}\right)$, as a function of $z_{0}$.

independent on the mobility, hence valid for both electrons and holes.

\section{Simulation}

Complementary to the experiments, a Monte Carlo simulation of the charge cylinder expansion was performed with a software tool specifically designed for modeling the physics of direct conversion detectors [16]. The basic simulation steps involve (a) the generation creation of electron/hole pairs along the pion track, (b) the three-dimensional drift and diffusion movement of the charges within a custom-defined electric field, and (c) the tracking of the positions where the holes finally reach the cathode plane of the detector, resulting in a two-dimensional collected charge density map on the cathode plane.

The basic drift/diffusion model described in [16] was extended by a Coulomb repulsion model which uses an algorithm of Barnes and Hat [17] to enable the computation of multi particle physics in a reasonable time.

The charge tracking was performed in a Cartesian coordinate system in which a silicon slice of $300 \mu \mathrm{m}$ thickness (representing the detector) is placed parallel to the $x-y-p l a n e$. The anode plane is placed at $z=0 \mu \mathrm{m}$ and the cathode plane is placed at $z=300 \mu \mathrm{m}$. The pion beam is directed parallel to the y-axis for a number of custom heights in steps of $50 \mu \mathrm{m}$ between $z=50 \mu \mathrm{m}$ and $z=250 \mu \mathrm{m}$. For silicon, an electron mobility of $1415 \mathrm{~cm}^{2} /(\mathrm{Vs})$, a hole mobility of $480 \mathrm{~cm}^{2} /(\mathrm{Vs})$, and a relative permittivity of $\epsilon_{r}=11.86$ were used. The detector is biased by a voltage of $100 \mathrm{~V}$. A simulation was performed under the assumption of a homogeneous electric field but also for an inhomogeneous one-dimensional electric field from a TCAD-based finite element calculation of the internal polarization (see Fig. 15). The space charge used for the calculation was $5 \times 10^{11} \mathrm{~cm}^{-3}$, corresponding to a depletion voltage of $34 \mathrm{~V}$ for a $300 \mu \mathrm{m}$ thick silicon sensor.

The initial charge generation creation was emulated by the random distribution of electron/hole pairs in a y-oriented cylinder of $40 \mathrm{~nm}$ diameter and of about $1 \mathrm{~mm}$ axial length and generated from the conversion of a $198 \mathrm{eV} / \mu \mathrm{m}$ average energy deposition within the simulated cylinder volume, with a

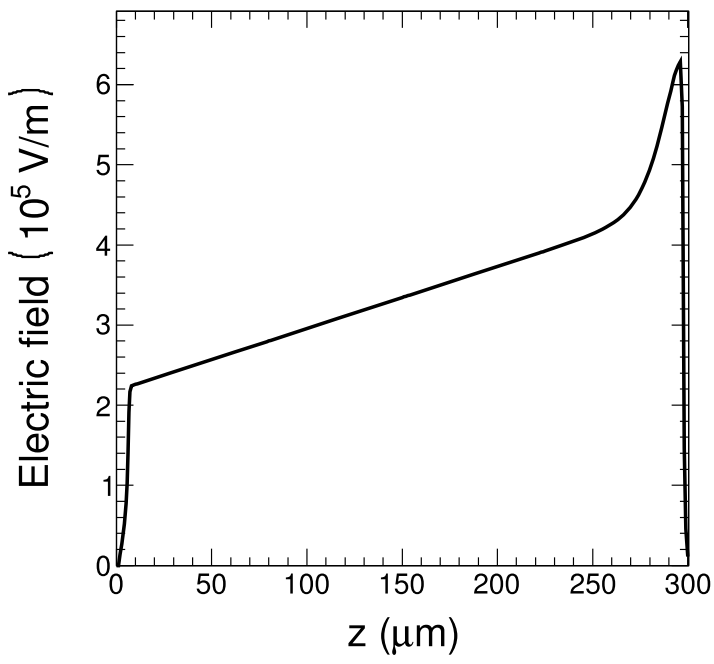

Fig. 15. Electric field strength along the z-depth-axis.

constant of $3.6 \mathrm{eV}$ per electron/hole pair, thus reproducing our measured carriers density of $55 \mu \mathrm{m}^{-1}$.

The simulation result is a collected charge distribution map showing an almost constant density along the y-axis (within the $y$-limits of the initially cylindrical charge creation volume) and a Gaussian function-like profile along the x-axis. In further processing, the charge density is averaged along the y-axis, and the resulting one-dimensional charge profile along the $\mathrm{x}$-axis is fitted by a Gaussian function from which the sigma value is taken for comparison with the experiment. Our simulation tool also allows to separate the contributions from the diffusion process only and from the Coulomb repulsion only. The theoretical pure Coulomb expansion predicts a homogeneous cylindrical cloud with outer radius $r_{0}$. For comparison with the diffusion process, an equivalent radius $r_{e q}$ has been computed as the one at which the Coulomb cloud contains the same amount of charge as a one-sigma diffusion cloud. The result is, as shown in Fig. 16, where the simulated points are superimposed to the data points. The two simulations regimes, the one based on a homogeneous electric field and the one based on the custom field from the TCAD calculation are both displayed. For the homogeneous field case, the two separate contributions from diffusion and Coulomb repulsion are drawn, this latter in terms of $r_{e q}$. For an immediate comparison between data and simulation, a linear fit to the data points is shown as well. Although the overall trend seems to be reproduced, the simulation results appear to be systematically lower than the data. Possible causes of this discrepancy are discussed in Section IX.

\section{Generalization of the Result}

The $\sigma\left(z_{0}\right)$ function as determined from the previous analysis represents the charge cloud Gaussian width at $z=300 \mu \mathrm{m}$ (i.e., at the collection electrode), when a particle traverses the sensor and generates free charge carriers at a depth $z_{0}$. We believe this result to be general, namely that $\sigma\left(z_{0}\right)$ is also usable to define a function $\sigma(z)$ describing the development of the charge cloud created by any particle interaction, where now $z$ represents the distance in depth traveled by the charge carriers from the point of initiation of the charge cloud. 


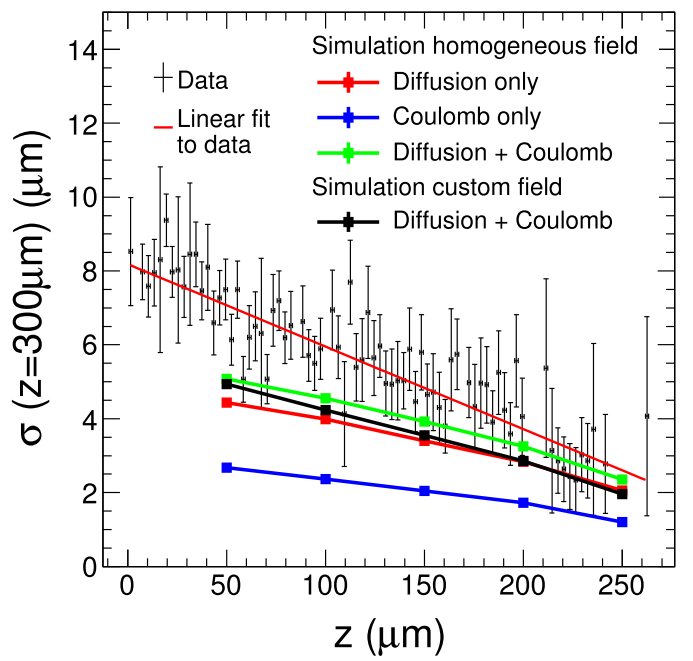

Fig. 16. Comparison of experimentally and by simulation acquired Gaussian sigma values of the charge density profiles. The segments drawn between the simulated points are merely to guide the eye.

To confirm this hypothesis, we performed a calculation in which $\sigma\left(z_{0}\right)$ is used to compute the energy spectrum detected from a molybdenum X-ray fluorescence source. The result is shown to be comparable with the corresponding spectrum obtained from a measurement.

\section{A. Calculation of Charge Sharing in X-ray Detection}

Following [18], $\sigma(z)$ was used to compute geometrically the effect of charge sharing in the detection of X-ray photons. Given a photon of energy $E_{0}$ that is converted at position $\left(x_{0}, y_{0}, z_{0}\right)$ in the sensor, the energy (more precisely, the amount of charge) reaching $z=300 \mu \mathrm{m}$ (i.e., the collection electrode) is distributed on the $x, y$ plane according to the Gaussian profile

$$
\frac{d^{2} E}{d x d y}\left(x, y ; x_{0}, y_{0}, z_{0}\right)=\frac{E_{0}}{\sqrt{2 \pi} \sigma\left(z_{0}\right)} e^{-\frac{\left(x-x_{0}\right)^{2}+\left(y-y_{0}^{2}\right)}{2 \sigma^{2}\left(z_{0}\right)}} .
$$

Here again only pure diffusion is considered. The energy detected by the pixel is given by

$$
E\left(x_{0}, y_{0}, z_{0}\right)=\int_{\text {Pixel area }} \frac{d^{2} E}{d x d y}\left(x, y ; x_{0}, y_{0}, z_{0}\right) d x d y .
$$

Fig. 17 shows an example of the detected energy fractions calculated with equation (17), for $z_{0}=7 \mu \mathrm{m}$. Similar calculations can be performed for any value of $z_{0}$.

The probability for a photon of energy $E_{0}$ to interact at a given depth $z_{0}$ in a sensor of thickness $t$ is given in terms of the exponential attenuation law

$$
P\left(z_{0}, E_{0}\right)=\frac{e^{-\mu\left(E_{0}\right) z_{0}}}{\int_{0}^{t} e^{-\mu\left(E_{0}\right) t^{\prime}} d t^{\prime}},
$$

where $\mu\left(E_{0}\right)$ is the energy dependent attenuation coefficient for the sensor material. Combining equation (18) and equation (17), it is possible to calculate the effect of the detector energy response on the detection of a spectrum $S\left(E_{0}\right)$.

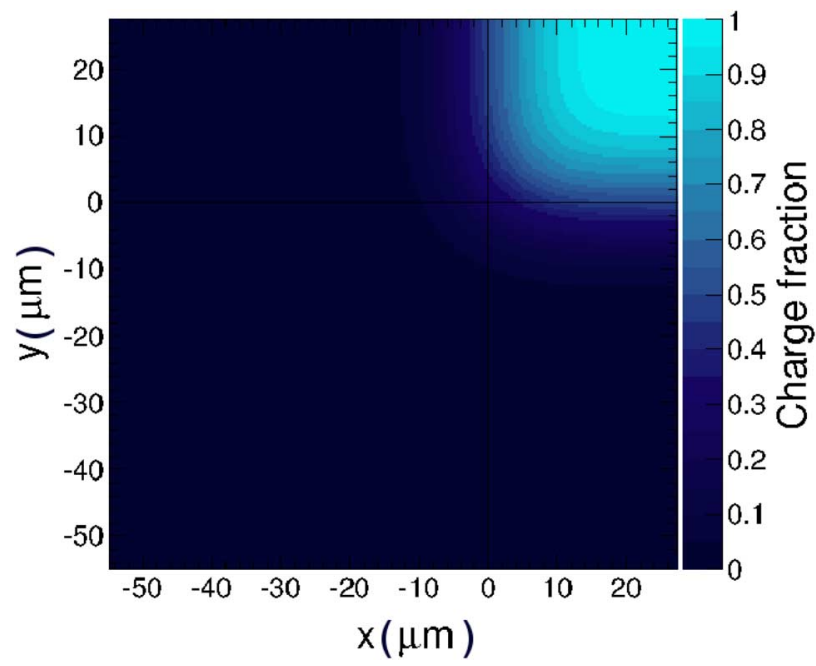

Fig. 17. Application of equation (17) using $\sigma(z)$ as determined from the test beam data. The color scale represents the energy fraction seen by the pixel at the top right corner (by symmetry, only one quarter of it is represented).

\section{B. Measurement and Comparison with the Model}

The spectrum used for the test is taken from the X-ray fluorescence emission of a molybdenum target irradiated by the primary beam of a tungsten anode X-ray tube. The cumulative spectrum was measured by varying the threshold of the Timepix detector while exposing it to the secondary radiation. Fitting the threshold scan with the method described in [19] allows to retrieve the two components of the source spectrum $S\left(E_{0}\right)$ $\left(K_{\alpha}=17.5 \mathrm{keV}, K_{\beta}=19.6 \mathrm{keV}\right.$, width $\left.0.6 \mathrm{keV}\right)$. The energy response of the Timepix detector is then applied on $S\left(E_{0}\right)$ using equations (17) and (18), and $\sigma(z)$ as determined from the test beam data analysis. The $\sigma(z)$ function was parametrized by fitting a straight line to the data in Fig. 13. This choice is not driven by any theoretical modelization, but is taken as a surrogate description of the data. The computed spectrum is then compared to the measured one, obtained by taking the derivative of the threshold scan.

The calculated spectrum at first does not reproduce the data satisfactorily. What we observe is that, in order to adapt the model to the data, the $\sigma(z)$ function has to be shifted. A fairly small offset has to be introduced in the linear parametrization. Indeed, we find it reasonable to assume that the initial size of the charge cloud, which determines the offset of $\sigma(z)$, has to be adapted from case to case. Moreover, the initial size of a single ionization cluster in the detection of a charged particle is different from the one in X-ray detection. While a charged particle creates a trail of low energy electrons (hundreds of eV) along its path, X-ray photons create a single medium energy photo-electron (keV's). For this measurement, the optimization of the $\sigma(z)$ offset was done by minimizing the root mean square distance between the data and the model. The final result is shown in Fig. 18 , obtained by shifting $\sigma(z)$ by $\Delta \sigma=(0.49 \pm 0.21) \mu \mathrm{m}$.

\section{DISCUSSION}

The analysis described in this paper shows how to measure the charge cloud profile development while the carriers are 


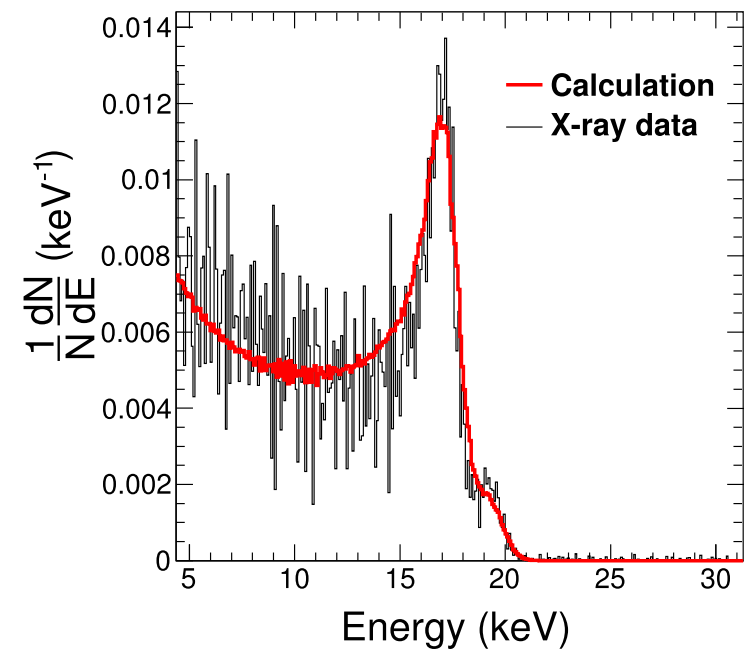

Fig. 18. Result of the X-ray test. The molybdenum fluorescence spectrum as calculated using $\sigma(z)$ (solid line) is well reproducing the spectrum measured by the Timepix detector. This latter arises from single pixels raw data, without any clustering algorithm applied.

drifting through a fully depleted silicon sensor from a well-defined space point of generation under the influence of the electric field. The result displayed in Fig. 13 shows that, within the experimental uncertainties, this trend is well described by a linear model. The $\sqrt{\text { time }}$ dependency of diffusion is not visible because the profile evolution is studied as a function of the drift distance, and not of the drift time.

On first approximation, the two simulation studies confirm this conclusion, at least in shape, while we still don't find a perfect agreement on absolute values with the data. The discrepancy with respect to the data is most likely to be attributed to the fact that (a) the electronic response of the readout chip is not accounted for and (b) the electric field considered is only one-dimensional. This latter point means that distortions of the field in the proximity of the inter-pixel gaps and the readout electrodes are not considered, whereas these are expected to also affect the charge collection properties of the sensor. Furthermore, in our simulation, we assumed a constant hole mobility, while a field-dependent mobility might improve the results.

In comparison with earlier studies of signal charge generation and carrier diffusion, the unique point of this work is the possibility to determine charge diffusion and effective signals on the relevant contacts, starting from basically any point inside the bulk volume of the sensor. Using a high energy particle beam allows, contrary to the narrow X-ray beam method presented in [4], to study all pixels simultaneously. Also, the depth of conversion for X-ray photons is known statistically and not for individual photons, while in our method we extract precise position information on the single detected particles.

A conventional pulse-height based technique for the determination of transport parameters in silicon was presented in the $80 \mathrm{~s}$ by Belau et al. [20] using measurements on silicon microstrip detectors, from which they could extract the final shape of the charge cloud profile. Much earlier, Jacoboni et al. [21] provided an extensive review on measurements of the charge transport parameters of silicon and germanium and their values for mobility and diffusion constants are usually quoted in handbooks. While for contemporary silicon and germanium this may not present an issue, these measurements all used charge generation at or near the surfaces of the semiconductor samples, which may not be optimal for all semiconductors.

Our statistics, while considerable, does not yet allow to determine the mean signal for each space point individually, for example in microscopic volumes of $3 \times 3 \times 3 \mu \mathrm{m}^{3}$, and here many points have been combined into single, averaged data sets. But the method described could be applied in this much more extended characterization, if somewhat more data would be collected, not even in very long exposures. It has to be admitted that a penetrating beam of energetic, minimum ionizing particles is required, for which there is limited availability worldwide. However, the more recent Timepix3+SPIDR readout system [10] would operate in beam intensities up to $10^{5} \mathrm{~s}^{-1}$, allowing massive data taking in a short time. An interesting extension would then be for semiconductors other than silicon, such as $\mathrm{CZT}, \mathrm{CdTe}$, or $\mathrm{HgI}_{2}$, for which indeed a 3D mapping of signal losses could be made. More work would be needed to determine if such a mapping would be stable with time and also be temperature-independent. Using a position-sensitive readout such as Timepix would allow to complement studies such as the ones presented in [22]. A full map of the diffusion profiles across the sensor volume could be built using our method with a beam of charged particles, and the effect of localized Te inclusions on the transport properties of the crystal could be observed directly.

\section{Conclusion And Outlook}

The necessity to characterize the transport properties of our sensor originated from the need to determine the energy response of a silicon Timepix-readout-based detector prototype for X-ray imaging applications. The results obtained in this study have already been successfully applied in a numerical framework for the calculation of the response function [13] and it is shown that the method complements the approach of modeling of the detector response using simulations. However, we believe that our study can be also interpreted in a more general setting.

Using the grazing angle method, one can benefit from the high-precision particle tracking capability of a radiation imaging semiconductor detector, not necessarily silicon, with a segmented contact matrix in connection with an advanced, spectroscopic pixel readout chip, like Timepix, to retrieve detailed information on the signal charge generation and the development of the charge distribution as a function of time, for space points in the bulk of the 3-dimensional sensitive volume, with precision well below the pixel dimensions themselves, and without the need to provide a priori parameters. Combined with the analogue/digital pulse height measurements at single pixel level, implemented in Timepix, eventually more precise measurements of transport properties might be achieved for a variety of semiconductor detector materials. Ultimately, it might even become possible to also characterize individually sensors with bulk defects and non-homogeneous charge collection properties. It can be expected that much more work will be done in the future on binary semiconductor materials, and the method described would present a possible approach for detailed characterization, even deep inside the bulk. So 
far, hardly any non-destructive alternative exists for such bulk analysis.

\section{REFERENCES}

[1] X. Llopart, R. Ballabriga, M. Campbell, L. Tlustos, and W. Wong, "Timepix, a 65k programmable pixel readout chip for arrival time, energy and/or photon counting measurements," Nucl. Instrum. Meth. A, vol. 585, pp. 106-108, 2008, Erratum.

[2] M. Campbell, E. Heijne, T. Holy, J. Jakubek, J. Idarraga, and C. Lebel et al., "Study of charge sharing in a silicon pixel detector by means of $\alpha$-particles interacting with a Medipix2 device," Nucl. Instrum. Meth. $A$, vol. 591, pp. 38-41, 2008.

[3] J. Bouchami, A. Gutierrez, A. Houdayer, J. Jakubek, C. Lebel, and C. Leroy et al., "Study of charge sharing in a silicon pixel detector with heavy ionizing particles interacting with a medipix2 device," Nucl. Instrum. Meth. A, vol. 607, pp. 196-198, 2009.

[4] J. Jakubek, "Energy sensitive x-ray radiography and charge sharing effects in pixelated detector," Nucl. Instrum. Meth. A, vol. 607, pp. 192-195, 2009.

[5] M. Sinor, J. Jakubek, V. Linhart, B. Mikulec, S. Pospisil, and B. Sopko, "Charge sharing studies with a medipix1 pixel device," Nucl. Instrum. Meth. A, vol. 509, pp. 346-354, 2003.

[6] B. Norlin, C. Fröjdh, H.-E. Nilsson, H. Graafsma, V. Vonk, and C. Ponchut, "Characterization of the charge sharing in pixellated Si detectors with single-photon processing readout," Nucl. Instrum. Meth. $A$, vol. 563, pp. 133-136, 2006.

[7] J. Visser, B. van der Heijden, S. J. A. Weijers, R. de Vries, and J. L. Visschers, "A gigabit per second read-out system for medipix quads," Nucl. Instrum. Meth. A, vol. 633, pp. S22-S25, 2011.

[8] D. Krapohl, C. Fröjdh, E. Fröjdh, E. N. Gimenez, D. Maneuski, and V. O'Shea et al., "Comparison of energy resolution spectra of CdTe TIMEPIX detector working in photon counting and time-over-threshold mode," in Proc. IEEE Nucl. Sci. Symp. Conf. Rec., Oct. 27-Nov., 2, 2013, pp. 1-4.

[9] A. P. Butler, P. H. Butler, S. T. Bell, G. A. Chelkov, D. V. Dedovich, and M. A. Demichev et al., "Measurement of the energy resolution and calibration of hybrid pixel detectors with GaAs:Cr sensor and Timepix readout chip," arXiv:1501.03334.
[10] T. Poikela, J. Plosila, T. Westerlund, M. Campbell, M. De Gaspari, and X. Llopart, "Timepix3: A $65 \mathrm{~K}$ channel hybrid pixel readout chip with simultaneous ToA/ToT and sparse readout," J. Instrum., vol. 9, p. C05013, 2014

[11] J. Jakubek, "Precise energy calibration of pixel detector working in time-over-threshold mode," Nucl. Instrum. Meth. A, vol. 633, pp. S262-S266, 2011.

[12] J. Beringer, J.-F. Arguin, R. M. Barnett, K. Copic, O. Dahl, and D. E. Groom et al., "Particle data group," Phys. Rev., vol. D86, p. 010001, 2012.

[13] E. J. Schioppa, D. Banerjee, J. Visser, T. Klaver, E. Koffeman, and W. Bras, "Measurement of the energy response function of a silicon pixel detector readout by a timepix chip using synchrotron radiation," J. Instrum., vol. 9, p. 08002, 2014.

[14] G. Hall, "Ionization energy losses of highly relativistic charged particles in thin silicon layers," Nucl. Instrum. Meth. A, vol. 220, pp. 356-362, 1984.

[15] S. Hancock, F. James, J. Movchet, P. G. Rancoita, and L. Van Rossum, "Energy loss and energy straggling of protons and pions in the momentum range $0.7 \mathrm{GeV} / \mathrm{c}$ to $115 \mathrm{GeV} / \mathrm{c}$," Phys. Rev., vol. A28, no. 615, pp. 356-362, 1983.

[16] K. J. Engel, R. Steadman, and C. Hermann, "Pulse temporal splitting in photon counting x-ray detectors," IEEE Trans. Nucl. Sci., vol. 59, pp. 1480-1490, 2012.

[17] J. Barnes and P. Hut, "A hierarchical $\mathrm{O}(\mathrm{N} \log \mathrm{N})$ force-calculation algorithm," Nature, vol. 324, pp. 446-449, 1986.

[18] J. Uher, J. Jakubek, S. Mayo, A. Stevenson, and J. Tickner, "X-ray beam hardening based material recognition in micro-imaging," $J$. Instrum., vol. 6, 2011

[19] E. J. Schioppa, J. Uher, and J. Visser, "Construction and test of an $\mathrm{X}$-ray CT setup for material resolved 3D imaging with medipix based detectors," J. Instrum., vol. 7, 2012

[20] E. Belau, R. Klanner, G. Lutz, E. Neugebauer, H. J. Seebrunner, and A. Wylie et al., "Charge collection in silicon strip detectors," Nucl. Instrum. Meth. A, vol. 214, pp. 253-260, 1983.

[21] C. Jacoboni, C. Canali, G. Ottaviani, and A. Alberigi Quaranta, "A review of some charge transport properties of silicon," Solid-State Electron., vol. 20, pp. 77-89, 1977.

[22] A. E. Bolotnikov, N. M. Abdul-Jabbar, O. S. Babalola, G. S. Camarda, Y. Cui, and A. M. Hossain et al., "Effects of te inclusions on the performance of CdZnTe radiation detectors," IEEE Trans. Nucl. Sci., vol. 55 , no. 2 , pp. $2757-2764,2008$ 